\title{
Classification with Label Distribution Learning
}

\author{
Jing Wang and Xin Geng * \\ MOE Key Laboratory of Computer Network and Information Integration \\ School of Computer Science and Engineering, Southeast University, Nanjing 210096, China
}

\{wangjing91,xgeng\}@seu.edu.cn

\begin{abstract}
Label Distribution Learning (LDL) is a novel learning paradigm, aim of which is to minimize the distance between the model output and the groundtruth label distribution. We notice that, in real-word applications, the learned label distribution model is generally treated as a classification model, with the label corresponding to the highest model output as the predicted label, which unfortunately prompts an inconsistency between the training phrase and the test phrase. To solve the inconsistency, we propose in this paper a new Label Distribution Learning algorithm for Classification (LDL4C). Firstly, instead of KL-divergence, absolute loss is applied as the measure for LDL4C. Secondly, samples are reweighted with information entropy. Thirdly, large margin classifier is adapted to boost discrimination precision. We then reveal that theoretically LDL4C seeks a balance between generalization and discrimination. Finally, we compare LDL4C with existing LDL algorithms on 17 real-word datasets, and experimental results demonstrate the effectiveness of LDL4C in classification.
\end{abstract}

\section{Introduction}

Learning with ambiguity, especially label ambiguity [Gao et al., 2017], has become one of the hot topics among the machine learning communities. Traditional supervised learning paradigms include single-label learning (SLL) and multilabel learning (MLL) [Zhang and Zhou, 2014], which label each instance with one or multiple labels. MLL assumes that each instance is associated with multiple labels, which compared with SLL takes the label ambiguity into consideration somewhat. Essentially, both SLL and MLL consider the relation between instance and label to be binary, i.e., whether or not the label is relevant with the instance. However, there are a variety of real-world tasks that instances are involved with labels with different importance degree, e.g., image annotation [Zhou and Zhang, 2006], emotion recognition [Zhou et al., 2015], age estimation [Geng et al., 2013]. Consequently,

\footnotetext{
${ }^{*}$ Corresponding author.
}

a soft label instead of a hard one seems to be a reasonable solution. Inspired by this, recently a novel learning paradigm, called Label Distribution Learning (LDL) [Geng, 2016], is proposed. LDL tackles label ambiguity with the definition of label description degree. Formally speaking, given an instance $\mathbf{x}$, LDL assigns each $y \in \mathcal{Y}$ a real value $d_{\mathbf{x}}^{y}$ (label description degree), which indicates the importance of $y$ to $\mathbf{x}$. To make the definition proper, [Geng, 2016] suggests that $d_{\mathbf{x}}^{y} \in[0,1]$ and $\sum_{y \in \mathcal{Y}} d_{\mathbf{x}}^{y}=1$, and the real value function $d$ is called the label distribution function. Since LDL extends the supervision from binary to label distribution, which is more applicable for real-world scenarios.

Due to the utility of dealing with ambiguity explicitly, LDL has been extensively applied in varieties of real-world problems. According to the source of label distribution, applications can be mainly classified into three classes. The first one includes emotion recognition [Zhou et al., 2015], pre-release rating prediction on movies [Geng and Hou, 2015], et. al, where the label distribution is from the data. Applications of the second class include head pose estimation [Huo and Geng, 2017], crowd counting [Zhang et al., 2015], et. al, label distribution of which are generated by pre-knowledge. Representative applications of the third one include beauty sensing [Ren and Geng, 2017], label enhancement [Xu et al., 2018], where the label distribution is learned from the data. Notice that the aforementioned applications of LDL fall into the scope of classification, and we find that a learned LDL model is generally treated as a classification model, with the label corresponding to the highest model output as the prediction, which unfortunately draws an inconsistency between the aim of the training phrase and the goal of test phrase of LDL. In the training phrase, the aim of LDL is to minimize the distance between the model output and the ground-truth label distribution, while in the test phrase, the object is to minimize the $0 / 1$ error.

We tackle the inconsistency in this paper. In order to alleviate the inconsistency, we come up with three improvements, i.e., absolute loss, re-weighting with entropy information, and large margin. The proposal of applying absolute loss and introducing large margin are inspired by theory arguments, and re-weighting samples with information entropy is based on observing the gap between the metric of LDL and that of the corresponding classification model. Since improvements are mainly originated from well-established 
theory tools, we are able to provide theoretical analysis of the proposed method. Consequently, the proposed algorithm LDL4C is well-designed and theoretically sound.

The main contributions of this paper are summarized as followings,

1) We propose three key components for improving classification performance for LDL, i.e, absolute loss, reweighting samples with information entropy, and large margin.

2) We establish upper bounds for 0/1 error and error probability of the proposed algorithms. Theoretical and empirical studies show that LDL4C enjoys generalization and discrimination.

The rest of the paper is organized as follows. Firstly, related works are briefly reviewed. Secondly details of the proposed method are presented. Then we give the theoretical analysis of the proposed method. Next experimental results are reported. Finally, we conclude the paper.

\section{Related Work}

Existing studies on LDL are primarily concentrated on designing new algorithms for LDL, and many algorithms have already been designed for LDL. [Geng, 2016] suggests three strategies for designing LDL algorithms. The first one is Problem Transformation (PT), which generates SL dataset according to the label distribution and then learns the transformed dataset with SL algorithms. Algorithms of the first strategy include PT-SVM and PT-Bayes, which apply SVM and Bayes classifier respectively. The second one is Algorithm Adaptation (AA), algorithms of which adapt existing learning algorithms to deal with label distribution straightly. Two representative algorithms are AA- $k \mathrm{NN}$ and AA-BP. For $\mathrm{AA}-k \mathrm{NN}$, mean of label distribution of $k$ nearest neighbors is calculated as the predicted label distribution, and for AA-BP, one-hidden-layer neural network with multi-output is adapted to minimize the sum-squared loss of output of the network compared with the ground-truth label distribution. The last one is Specialized Algorithm (SA). This category of algorithms take characteristics of LDL into consideration. Two representative approaches of SA are IIS-LDL and BFGSLDL, which apply the maximum entropy model to learn the label distribution. Besides, [Geng and Hou, 2015] treats LDL as a regression problem, and proposes LDL-SVR, which embraces SVR to deal with the label distribution. Furthermore, [Shen et al., 2017] proposes LDLF, which extends random forest to learn label distribution. In addition, [Gao et al., 2017] provides the first deep LDL model DLDL. Notice that compared with the classic LDL algorithms, LDLF and DLDL support end-to-end training, which are suitable for computer vision tasks. Notice that none of aforementioned algorithms ever consider the inconsistency as we previously discussed.

There are few work on inconsistency between the training and the test phrase of LDL. [Gao et al., 2018] firstly recognizes the inconsistency in the application of age estimation, and designs a lightweight network to jointly learn the age distribution and the ground-truth age to bridge the gap. However the method is only suitable for real-valued label space and no theory guarantee is provided. Besides, one recent work [Wang and Geng, 2019] provides a theoretical analysis of the relation between the risk of LDL (absolute loss) and error probability of the corresponding classification function, discovering that LDL with absolute loss dominates classification. However, the major motivation of the paper is to understanding LDL theoretically, and no algorithm design to improve classification precision is provided.

\section{The Proposed Method}

\subsection{Preliminary}

Let $\mathcal{X}$ be the input space, and $\mathcal{Y}=\left\{y_{1}, y_{2}, \ldots, y_{m}\right\}$ be the label space. Denote the instance by $\mathbf{x}$. The label distribution function $d \in \mathbb{R}^{\mathcal{X} \times \mathcal{Y}}$ is defined as $d: \mathcal{X} \times \mathcal{Y} \mapsto \mathbb{R}$, satisfying the constraints $d_{\mathbf{x}}^{y}>0$ and $\sum_{y \in \mathcal{Y}} d_{\mathbf{x}}^{y}=1$, where $d_{\mathbf{x}}^{y}=d(\mathbf{x}, y)$ for convenience of notations. Given a training set $S=\left\{\left(\mathbf{x}_{1},\left[d_{\mathbf{x}_{1}}^{y_{1}}, \ldots, d_{\mathbf{x}_{1}}^{y_{m}}\right]\right), \ldots,\left(\mathbf{x}_{n},\left[d_{\mathbf{x}_{n}}^{y_{1}}, \ldots, d_{\mathbf{x}_{n}}^{y_{m}}\right]\right)\right\}$, the goal of LDL is to learn the unknown function $d$, and the object of LDL4C is to make a prediction.

\subsection{Classification via LDL}

In real-world applications we generally regard a learned LDL model as a classification mode. Formally speaking, denote the learned LDL function by $h$ and the corresponding classification function by $\hat{h}$, for a given instance $\mathbf{x}$, then we have

$$
\hat{h}(\mathbf{x})=\arg \max _{y \in \mathcal{Y}} h_{\mathbf{x}}^{y}
$$

i.e, the prediction is the label with the highest output. Intuitively if $h$ is near the ground-truth label distribution function $d$, then the corresponding classification function $\hat{h}$ is close to the Bayes classification (we assume $d$ is the conditional probability distribution function), thereby LDL is definitely related with classification. The mathematical tool which quantifies the relation between LDL and classification is the plugin decision theorem [Devroye et al., 1996]. Let $h^{*}$ be the Bayes classification function, i.e.,

$$
h^{*}(\mathbf{x})=\arg \max _{y \in \mathcal{Y}} d_{\mathbf{x}}^{y}
$$

then we have

Theorem 1. [Devroye et al., 1996; Wang and Geng, 2019] The error probability difference between $\hat{h}$ and $h^{*}$ satisfies

$$
\mathbb{P}(\hat{h}(\mathbf{x}) \neq y)-\mathbb{P}\left(h^{*}(\mathbf{x}) \neq y\right) \leq \mathbb{E}_{\mathbf{x}}\left[\sum_{y \in \mathcal{Y}}\left|h_{\mathbf{x}}^{y}-d_{\mathbf{x}}^{y}\right|\right] .
$$

The theorem says that if $h$ is close to $d$ in terms of absolute loss, then error probability of $\hat{h}$ is close to that of $h^{*}$. Theoretically LDL with absolute loss is directly relevant with classification. Also absolute loss is tighter than KL-divergence, since $|\mathbf{p}-\mathbf{q}| \leq 2 \sqrt{\mathrm{KL}(\mathbf{p}, \mathbf{q})}$, for $\mathbf{p}, \mathbf{q} \in \mathbb{R}^{m}$ [Cover and Thomas, 2012]. Accordingly we propose to use absolute loss in LDL for classification, instead of KL-divergence. Similar with [Geng, 2016], we use maximum entropy model to learn the label distribution. Maximum entropy mode is defined as

$$
h_{\mathbf{x}}^{y_{j}}=\frac{1}{Z} \exp \left(\mathbf{w}_{j} \cdot \mathbf{x}\right)
$$




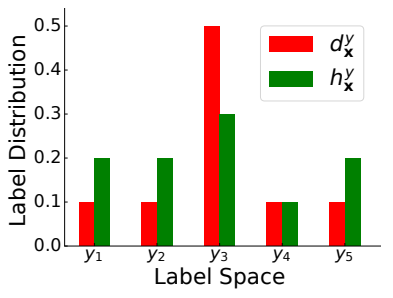

(a) $\ell_{1}=0.5, \ell_{\mathrm{ep}}=0.5$

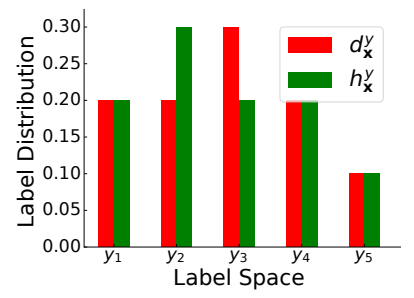

(b) $\ell_{1}=0.2, \ell_{\mathrm{ep}}=0.8$
Figure 1: An example to illustrate the gap between absolute loss for LDL and error probability for classification. The red bar represents the ground-truth label distribution, and the green one represents the learned label distribution.

where $Z=\sum_{j} \exp \left(\mathbf{w}_{j} \cdot \mathbf{x}\right)$ is the normalization factor. Absolute loss is applied as the measure for LDL, and consequently the problem can be formulated as

$$
\min _{\mathbf{W}} \sum_{i=1}^{n} \sum_{j=1}^{m}\left|h_{\mathbf{x}_{i}}^{y_{j}}-d_{\mathbf{x}_{i}}^{y_{j}}\right|+\frac{C}{2} \sum_{j=1}^{m}\left\|\mathbf{w}_{j}\right\|^{2}
$$

where $\mathbf{W}=\left[\mathbf{w}_{1}, \mathbf{w}_{2}, \ldots, \mathbf{w}_{m}\right]$ is the set of parameters.

\subsection{Re-weighting with Information Entropy}

After taking a second look at the Theorem (1), we will find out that model (2) actually optimizes the upper bound for error probability. This really brings a gap between absolute loss for LDL and 0/1 loss for classification. Fig. 1 is an illustrative example to demonstrate the gap, where $\ell_{1}$ denotes absolute loss and $\ell_{\text {ep }}$ denotes error probability defined as Eq. (15). As we can see from the figure, (b) is superior to (a) from the perspective of absolute loss. However (b) is inferior to (a) in terms of error probability. For (a), $y_{3}$ has the highest label distribution both for $d$ and $h$, thus prediction of $\hat{h}$ coincides with that of the Bayes classification function $h^{*}$. For (b), $y_{3}$ has the highest label distribution for $d$ and $y_{2}$ has the highest label distribution for $h$, thus the corresponding classification result is different with that of the Bayes classification. Essentially for label distribution which is multimodal (like (b) of Fig.1), the corresponding classification function is much more sensitive to the loss of LDL. While, for label distribution which is unimodal (like (a) of Fig.1), there is more room to manoeuvre. Information entropy seems to be a reasonable tool to quantize this sensitiveness, since a multimodal distribution generally brings higher information entropy compared with a unimodal one. Precisely, for the label distribution with higher information entropy (i.e., multimodal), the corresponding classification model is much more sensitive to the LDL loss, and vice verse. In other words, samples with high information entropy deserves more attention, which reminds us to re-weight samples with information entropy. Reweighted with information entropy will penalize large loss for samples with high information entropy, and leave more room for samples with low information entropy. Recall the definition of entropy information, for $\mathbf{x}$,

$$
E_{\mathbf{x}}=-\sum_{y \in \mathcal{Y}} d_{\mathbf{x}}^{y} \ln d_{\mathbf{x}}^{y}
$$

Accordingly the problems is then formulated as

$$
\min _{\mathbf{W}} \sum_{i=1}^{n} E_{\mathbf{x}_{i}} \sum_{j=1}^{m}\left|h_{\mathbf{x}_{i}}^{y_{j}}-d_{\mathbf{x}_{i}}^{y_{j}}\right|+\frac{C}{2} \sum_{j=1}^{m}\left\|\mathbf{w}_{j}\right\|^{2} .
$$

\subsection{LDL with Large Margin}

To further boost the classification precision, we borrow the margin theory. Formally speaking, let the model output corresponding to the Bayes prediction has a large margin over other labels, which pushes the corresponding classification model consistent with the Bayes classification model. Let $y_{i}^{*}$ be the Bayes prediction for $\mathbf{x}_{i}$, i.e., $y_{i}^{*}=h^{*}\left(\mathbf{x}_{i}\right)$. Then we assume a margin $\rho$ between $h_{\mathbf{x}_{i}}^{y_{i}^{*}}$ and $\max _{y \in\left\{\mathcal{Y}-y_{i}^{*}\right\}} h_{\mathbf{x}_{i}}^{y}$, and the problem can be formulated as

$$
\begin{array}{ll}
\min _{\mathbf{W}} & \sum_{i=1}^{n} E_{\mathbf{x}_{i}} \sum_{j=1}^{m}\left|h_{\mathbf{x}_{i}}^{y_{j}}-d_{\mathbf{x}_{i}}^{y_{j}}\right|+C_{1} \sum_{i=1}^{n} \xi_{i}+\frac{C_{2}}{2} \sum_{j=1}^{m}\left\|\mathbf{w}_{j}\right\|^{2}, \\
\text { s.t. : } \quad & h_{\mathbf{x}_{i}}^{y_{i}^{*}}-\max _{y \in\left\{\mathcal{Y}-y_{i}^{*}\right\}} h_{\mathbf{x}_{i}}^{y}>\rho-\xi_{i} \\
\quad \xi_{i} \geq 0, \quad \forall i \in[n],
\end{array}
$$

where $C_{1}, C_{2}$ are the balance coefficients for margin loss and regularization respectively. Large margin turns out to be a reasonable assumption since the ultimate goal of LDL4C is to pursuit the Bayes classifier. Overall, model (4) seeks a balance between LDL and large margin classifier.

\subsection{Optimization}

To start, for $\mathbf{x}$, define

$$
\alpha=h_{\mathbf{x}}^{y_{i}^{*}}-\max _{y \in\left\{\mathcal{Y}-y_{i}^{*}\right\}} h_{\mathbf{x}}^{y},
$$

and $\rho$-margin loss as $\ell^{\rho}(\alpha)=\max \left\{0,1-\frac{\alpha}{\rho}\right\}$. By introducing margin loss, model (4) can be re-formed as

$\min _{\mathbf{W}} \sum_{i=1}^{n} E_{\mathbf{x}_{i}} \sum_{j=1}^{m}\left|h_{\mathbf{x}_{i}}^{y_{j}}-d_{\mathbf{x}_{i}}^{y_{j}}\right|+C_{1} \sum_{i=1}^{n} \ell^{\rho}\left(\alpha_{i}\right)+\frac{C_{2}}{2} \sum_{j=1}^{m}\left\|\mathbf{w}_{j}\right\|^{2}$.

which can be optimized efficiently by gradient-based method ( $\ell^{\rho}$ is differential). We optimize the target function by BFGS [Nocedal and Wright, 2006]. Define $\phi$ as the step function,

$$
\phi(x)= \begin{cases}1 & \text { if } x \geq 0 \\ 0 & \text { otherwise }\end{cases}
$$

and denote the target function by $T$, gradient of which is obtained through

$$
\begin{aligned}
& \frac{\partial T}{\partial \mathbf{w}_{k}}=\sum_{i, j} E_{\mathbf{x}_{i}} \operatorname{sign}\left(h_{\mathbf{x}_{i}}^{y_{j}}-d_{\mathbf{x}_{i}}^{y_{j}}\right) \frac{\partial h_{\mathbf{x}_{i}}^{y_{j}}}{\mathbf{w}_{k}}+C_{2} \mathbf{w}_{k}+ \\
& C_{1} \sum_{i=1}^{n} \phi\left(\rho-\alpha_{i}\right)\left(\frac{\left.\left.\partial \max _{y \in\left\{\mathcal{Y}-y_{i}^{*}\right\}} h_{\mathbf{x}_{i}}^{y}\right)\right)}{\mathbf{w}_{k}}-\frac{\partial h_{\mathbf{x}_{i}}^{y_{i}^{*}}}{\mathbf{w}_{k}}\right) .
\end{aligned}
$$

Moreover, gradient of $h$ is got through

$$
\frac{\partial h_{\mathbf{x}}^{y_{j}}}{\partial \mathbf{w}_{k}}=\left[\mathbf{1}_{\{j=k\}} \frac{\exp \left(\mathbf{w}_{k} \cdot \mathbf{x}\right)}{\sum_{i} \exp \left(\mathbf{w}_{i} \cdot \mathbf{x}_{i}\right)}-\left(\frac{\exp \left(\mathbf{w}_{j} \cdot \mathbf{x}\right)}{\sum_{i} \exp \left(\mathbf{w}_{i} \cdot \mathbf{x}\right)}\right)^{2}\right] \mathbf{x}_{i} .
$$




\section{Theoretical Results}

In this section, we will demonstrate that, theoretically LDL4C seeks a trade-off between generalization and discrimination, where generalization is due to the LDL process, and discrimination is credited to the large margin classifier.

\subsection{Generalization}

Here by generalization we mean that the error probability of LDL4C converges to that of the Bayes classifier, by developing an upper bound for error probability of LDL4C. The basic steps are providing an upper bound for risk of LDL4C (with absolute loss) firstly, and then converting the risk bound into error probability bound by Theorem 1 .

Notice that maximum entropy model, i.e., Eq. (1) can be regarded as function combination of softmax function and multi-output linear regression function. Formally, let SF be the softmax function, $\mathcal{F}$ be a family of functions for multioutput linear regression. Denote the family of functions for the maximum entropy model by $\mathcal{H}$, then $\mathcal{H}=\{\mathbf{x} \mapsto$ SF $\circ f(\mathbf{x}): f \in \mathcal{F}\}$. To bound the risk of LDL4C, it suffices to derive an upper bound on the Rademacher complexity [Bartlett and Mendelson, 2003] of the model.

Theorem 2. Define $\mathcal{F}$ as $\mathcal{F}=\left\{\mathbf{x} \mapsto\left[\mathbf{w}_{1} \cdot \mathbf{x}, \ldots, \mathbf{w}_{m} \cdot \mathbf{x}\right]^{T}\right.$ : $\left.\left\|\mathbf{w}_{j}\right\| \leq 1\right\}$. Rademacher complexity of $\ell_{1} \circ \mathcal{H}$ satisfies

$$
\hat{\mathcal{R}}_{n}\left(\ell_{1} \circ \mathcal{H}\right) \leq \frac{2 \sqrt{2} m^{2} \max _{i \in[n]}\left\|\mathbf{x}_{i}\right\|_{2}}{\sqrt{n}} .
$$

Proof. Firstly, according to [Wang and Geng, 2019], $\ell_{1} \circ \mathrm{SF}$ is shown to be $2 m$-Lipschitz. And according to [Maurer, 2016], with $\ell_{1} \circ \mathrm{SF}$ being $2 m$-Lipschitz, then

$$
\hat{\mathcal{R}}_{n}\left(\ell_{1} \circ \mathcal{H}\right) \leq 2 \sqrt{2} m \sum_{j=1}^{m} \hat{\mathcal{R}}_{n}\left(\mathcal{F}_{i} \circ S\right),
$$

where $\mathcal{F}_{j}=\left\{\mathbf{x} \mapsto \mathbf{w}_{j} \cdot \mathbf{x}:\left\|\mathbf{w}_{j}\right\| \leq 1\right\}$. Moreover, according to [Kakade et al., 2009], Rademacher complexity of $\mathcal{F}_{j}$ satisfies

$$
\hat{\mathcal{R}}_{n}\left(\mathcal{F}_{j}\right) \leq \frac{\max _{i \in[n]}\left\|\mathbf{x}_{i}\right\|_{2}}{\sqrt{n}} .
$$

Finally substitute Eq. (9) into Eq. (8), and we finish the proof for Theorem (2).

Then data-dependent error probability for LDL4C is as following,

Theorem 3. Define $\hat{\mathcal{H}}=\left\{\mathbf{x} \mapsto \max _{y \in \mathcal{Y}} h_{\mathbf{x}}^{y}: h \in \mathcal{H}\right\}$. Then for any $\delta>0$, with probability at least $1-\delta$, for any $\hat{h} \in \hat{\mathcal{H}}$, such that

$$
\begin{array}{r}
\mathbb{P}(\hat{h}(\mathbf{x}) \neq y)- \\
\mathbb{P}\left(h^{*}(\mathbf{x}) \neq y\right) \leq \sum_{i}^{n} \sum_{j}^{m}\left|h_{\mathbf{x}_{i}}^{y_{j}}-d_{\mathbf{x}_{i}}^{y_{j}}\right|+ \\
\frac{\left.4 \sqrt{2} m^{2} \max _{i \in[n]}|| \mathbf{x}_{i}\right|_{2}}{\sqrt{n}}+6 \sqrt{\frac{\log 2 / \delta}{2 n}} .
\end{array}
$$

Proof. Firstly, according to [Bartlett and Mendelson, 2003; Mohri et al., 2012], and $\sum_{y}\left|d_{\mathbf{x}}^{y}-h_{\mathbf{x}}^{y}\right| \leq 2$ (triangle inequality), data-dependent risk bound for LDL4C is as

$$
\begin{array}{r}
\mathbb{E}_{\mathbf{x}}\left[\sum_{j=1}^{m}\left|h_{\mathbf{x}}^{y_{j}}-d_{\mathbf{x}}^{y_{j}}\right|\right] \leq \sum_{i}^{n} \sum_{j}^{m}\left|h_{\mathbf{x}_{i}}^{y_{j}}-d_{\mathbf{x}_{i}}^{y_{j}}\right|+ \\
\frac{\left.4 \sqrt{2} m^{2} \max _{i \in[n]}|| \mathbf{x}_{i}\right|_{2}}{\sqrt{n}}+6 \sqrt{\frac{\log 2 / \delta}{2 n}} .
\end{array}
$$

Secondly combine Theorem (1) and Eq. (3), which completes the proof for the theorem.

\subsection{Discrimination}

In this part, we will borrow margin theory to demonstrate that LDL4C enjoys discrimination. By discrimination, we mean the ability to output the same prediction as the Bayes prediction. According to [Bartlett and Mendelson, 2003], with margin loss, we have

Theorem 4. Define $\tilde{\mathcal{H}}$ as $\tilde{\mathcal{H}}=\left\{\left(\mathbf{x}, y^{*}\right) \mapsto h\left(\mathbf{x}, y^{*}\right)-\right.$ $\left.\max _{y \in\left\{\mathcal{Y}-y^{*}\right\}} h(\mathbf{x}, y): h \in \mathcal{H}\right\}$. Fix $\rho>0$. Then, for any $\delta>0$, with probability at least $1-\delta$, for all $h \in \mathcal{H}$, such that

$$
\mathbb{E}\left[\ell^{\rho}(\alpha)\right] \leq \sum_{i=1}^{n} \ell^{\rho}\left(\alpha_{i}\right)+2 \hat{\mathcal{R}}_{n}\left(\ell^{\rho} \circ \tilde{\mathcal{H}}\right)+3 \sqrt{\frac{\log 2 / \delta}{2 n}} .
$$

Since $\ell^{\rho}$ satisfies $1 / \rho$-Lipschitzness, we have $\hat{\mathcal{R}}_{n}\left(\ell^{\rho} \circ\right.$ $\tilde{\mathcal{H}}) \leq \frac{1}{\rho} \hat{\mathcal{R}}_{n}(\tilde{\mathcal{H}})$. And according to [Mohri et al., 2012], $\hat{\mathcal{R}}_{n}(\tilde{\mathcal{H}}) \leq 2 m \hat{\mathcal{R}}_{n}\left(\Pi_{1}(\mathcal{H})\right)$, where $\Pi_{1}(\mathcal{H})$ is defined as

$$
\Pi_{1}(\mathcal{H})=\{\mathbf{x} \mapsto h(\mathbf{x}, y): y \in \mathcal{Y}, h \in \mathcal{H}\} .
$$

Since $\mathbf{1}_{\left\{h(\mathbf{x}) \neq y^{*}\right\}}<\ell^{\rho}(\alpha)$, thus we have

$$
\mathbb{E}\left[\mathbf{1}_{h(\mathbf{x}) \neq y^{*}}\right] \leq \sum_{i=1}^{n} \ell^{\rho}\left(\alpha_{i}\right)+\frac{4 m \hat{\mathcal{R}}_{n}\left(\Pi_{1}(\mathcal{H})\right)}{\rho}+3 \sqrt{\frac{\log \frac{2}{\delta}}{2 n}} .
$$

And $\hat{\mathcal{R}}_{n}\left(\Pi_{1}(\mathcal{H})\right)$ can be bounded as

$$
\hat{\mathcal{R}}_{n}\left(\Pi_{1}(\mathcal{H})\right) \leq \frac{(m-1) \gamma \exp (\gamma)}{\sqrt{n}},
$$

where $\gamma=2 \max _{i \in[n]}\left\|\mathbf{x}_{i}\right\|$, which leads to

Theorem 5. Fix $\rho>0$. Then for any $\delta>0$, with probability at least $1-\delta$, for all $h \in \mathcal{H}$ such that

$\mathbb{E}\left[\mathbf{1}_{h(\mathbf{x}) \neq y^{*}}\right] \leq \sum_{i=1}^{n} \ell^{\rho}\left(\alpha_{i}\right)+\frac{4(m-1) m \gamma \exp (\gamma)}{\rho \sqrt{n}}+3 \sqrt{\frac{\log \frac{2}{\delta}}{2 n}}$.

\section{Experiments}

\subsection{Experimental Configuration}

Real-word datasets. The experiments are extensively conducted on seventeen datasets totally, among which fifteen are from [Geng, 2016], and $\mathrm{M}^{2} \mathrm{~B}$ is from [Nguyen et al., 2012], and SCUT-FBP is from [Xie et al., 2015]. Note that $\mathrm{M}^{2} \mathrm{~B}$ and SCUT-FBP are transformed to label distribution as [Ren and Geng, 2017]. Datasets are from a variety of fields, and statistics about the datasets are listed as Table 1. 
Proceedings of the Twenty-Eighth International Joint Conference on Artificial Intelligence (IJCAI-19)

\begin{tabular}{lrrr}
\hline Dataset & \# Examples & \# Features & \# Labels \\
\hline SJAFFE & 213 & 243 & 6 \\
M $^{2}$ B & 1240 & 250 & 5 \\
SCUT-FBP & 1500 & 300 & 5 \\
Natural Scene & 2000 & 294 & 9 \\
Yeast-alpha & 2465 & 24 & 18 \\
Yeast-cdc & 2465 & 24 & 15 \\
Yeast-cold & 2465 & 24 & 4 \\
Yeast-diau & 2465 & 24 & 7 \\
Yeast-dtt & 2465 & 24 & 4 \\
Yeast-elu & 2465 & 24 & 14 \\
Yeast-heat & 2465 & 24 & 6 \\
Yeast-spo & 2465 & 24 & 6 \\
Yeast-spo5 & 2465 & 24 & 3 \\
Yeast-spoem & 2465 & 24 & 2 \\
SBU_3DFE & 2500 & 243 & 6 \\
Movie & 7755 & 1869 & 5 \\
Human Gene & 17892 & 36 & 68 \\
\hline
\end{tabular}

\begin{tabular}{lrrrr}
\hline Dataset & LDL & LDL $+\ell_{1}$ & LDL+RW & LDL+LM \\
\hline SJAFFE & .437 & .417 & $\mathbf{. 3 9 4}$ & .426 \\
M $^{2}$ B & .486 & .486 & $\mathbf{. 4 8 2}$ & .485 \\
SCUT-FBP & .467 & .470 & $\mathbf{. 4 6 5}$ & .467 \\
Natural Scene & .597 & .420 & $\mathbf{. 4 2 1}$ & .425 \\
Yeast-alpha & .891 & $\mathbf{. 7 8 7}$ & $\mathbf{. 7 8 7}$ & $\mathbf{. 7 8 7}$ \\
Yeast-cdc & .823 & $\mathbf{. 8 2 0}$ & .821 & .821 \\
Yeast-cold & .580 & $\mathbf{. 5 7 6}$ & $\mathbf{. 5 7 6}$ & $\mathbf{. 5 7 6}$ \\
Yeast-diau & .694 & $\mathbf{. 6 6 5}$ & .668 & .669 \\
Yeast-dtt & .629 & .630 & $\mathbf{. 6 2 7}$ & $\mathbf{. 6 2 7}$ \\
Yeast-heat & .702 & .676 & $\mathbf{. 6 7 5}$ & .677 \\
Yeast-spo & .548 & $\mathbf{. 5 4 7}$ & $\mathbf{. 5 4 7}$ & $\mathbf{. 5 4 7}$ \\
Yeast-spoem & .435 & $\mathbf{. 4 0 0}$ & .416 & .422 \\
Movie & .416 & .416 & $\mathbf{. 4 1 0}$ & .414 \\
\hline
\end{tabular}

Table 2: Experimental results of comparing BFGS-LDL with BFGS$\mathrm{LDL}+\left\{\ell_{1}, \mathrm{RW}, \mathrm{LM}\right\}$ in terms of $0 / 1$ loss. The best results are highlighted in bold face.

Table 1: Statistics of 17 real-world datasets used in the experiments.

Comparing algorithms. We compare LDL4C with six popular LDL algorithms, i.e., PT-Bayes, PT-SVM, AABP, AA- $k$ NN, LDL-SVR [Geng and Hou, 2015], BFGSLDL, and two state-of-the-art LDL algorithms, i.e., StructRF [Chen et al., 2018] and LDL-SCL [Zheng et al., 2018]. For LDL4C, the balance parameter $C_{1}$ and $C_{2}$ are selected from $\{0.001,0.01,0.1,1,10,100\}$ and $\rho$ is chosen from $\{0.001,0.01,0.1\}$ by cross validation. Moreover for AA$\mathrm{BP}$, the number of hidden-layer neurons is set to 64 , and for AA- $k \mathrm{NN}$, the number of nearest neighbors $k$ is selected from $\{3,5,7,9,11\}$. Furthermore, for BFGS-LDL, we follow the same settings with [Geng, 2016]. Besides, the insensitive parameter $\epsilon$ is set to 0.1 as suggested by [Geng and Hou, 2015], and rbf kernel is used for both PT-SVM and LDLSVR. For StructRF, depth is set to 20 and number of trees is set to 50 , and sampling ratio is set to 0.8 as suggested by [Chen et al., 2018]. In addition, for LDL-SCL, $\lambda_{1}=0.001$, $\lambda_{2}=0.001, \lambda_{3}=0.001$ and number of clusters $m=6$ as given in [Zheng et al., 2018]. Finally, all algorithms are examined on 17 datasets with 10-fold cross validation, and average performance is reported.

Evaluation metrics. We test algorithms in terms of two measures, 0/1 loss and error probability. For 0/1 loss, we regard the label with the highest label distribution as the ground-truth label. Given an instance $\mathbf{x}$ with prediction $y$, the error probability is defined as

$$
\ell_{\mathrm{ep}}(\mathbf{x}, y)=1-d_{\mathbf{x}}^{y} \text {. }
$$

\subsection{Experimental Results}

Firstly, to validate the three key components (i.e., absolute loss, re-weighting with information entropy, and large margin) for boosting classification precision, we add each of the components into BFGS-LDL and compare 0/1 loss with BFGS-LDL. The performance is reported in Table 2. Due to the page limitation, we only report part of the results. Here "LDL" is short for BFGS-LDL. "LDL $+\ell_{1}$ " represents BFGSLDL with absolute loss, and "LDL+RW" represents BFGSLDL with information entropy re-weighting, and "LDL+LM" stands for BFGS-LDL with large margin. As we can see from the table, armed with three key components, the corresponding LDL model can achieve better classification performance

compared with the original BFGS-LDL model. Secondly we conduct extensive experiments of LDL4C and comparing methods on 17 real-world datasets in terms of 0/1 loss and error probability, and the experimental results are presented as in Table 3 and Table 4 respectively. The pairwise t-test at 0.05 significance level is conducted, with the best performance marked in bold face. From the tables, we can get that:

- In terms of 0/1 loss, LDL4C rank 1st among 13 datasets, and is significantly superior to other comparing methods in $70 \%$ cases, which validates the ability of LDL4C for performing classification.

- In terms of error probability, LDL4C ranks 1st among 12 datasets, and achieves significant better performance in $46 \%$ cases, which discloses that LDL4C has better generalization.

\section{Conclusions}

This paper tackles the inconsistency between the training and test phrase of LDL when applied in applications. In the training phrase of LDL, aim of which is to learn the given label distribution, i.e., minimizing (maximizing) distance (similarity) between the model output and the given label distribution. However, for applications of LDL, a learned LDL model is generally treated as a classification model, with the label corresponding to the highest output as the prediction. The proposed algorithm LDL4C alleviates the inconsistency with three key improvements. The first one is inspired by the plug-in decision Theorem 1, which states that absolute loss is directly related with classification error, thereby absolute loss is applied as the loss function for LDL4C. The second one stems from noticing the gap between absolute loss and the classification error, which suggests re-weighting samples with information entropy to pay more attention to multimodal distribution. The last one is due to margin theory, which introduces a margin between $h_{\mathbf{x}}^{y^{*}}$ and $\max _{y \in\left\{\mathcal{Y}-y^{*}\right\}} h_{\mathbf{x}}^{y}$ to enforce the corresponding classification model approaching the Bayes decision rule. Furthermore, we explore the effectiveness of LDL4C theoretically and empirically. Theoretically, LDL4C enjoy both generalization and discrimination, and empirically extensive comparing experiments clearly manifest the advantage of LDL4C in classification. 
Proceedings of the Twenty-Eighth International Joint Conference on Artificial Intelligence (IJCAI-19)

\begin{tabular}{|c|c|c|c|c|c|c|c|c|c|}
\hline Dataset & PT-Bayes & PT-SVM & AA-BP & $\mathrm{AA}-k \mathrm{NN}$ & LDL-SVR & StructRF & LDL-SCL & BFGS-LDL & LDL4C \\
\hline SJAFFE & $.783 \pm .016 \bullet$ & $.785 \pm .003 \bullet$ & $.807 \pm .003 \bullet$ & $.483 \pm .012 \bullet$ & $.686 \pm .004 \bullet$ & $.569 \pm .011 \bullet$ & $.789 \pm .002 \bullet$ & $.437 \pm .018$ & $.394 \pm .010$ \\
\hline $\mathrm{M}^{2} \mathrm{~B}$ & $.645 \pm .003 \bullet$ & $.552 \pm .003 \bullet$ & $.487 \pm .001$ & $.484 \pm .001$ & $.495 \pm .002$ & $.483 \pm .002$ & $.498 \pm .001$ & $.486 \pm .002$ & $.481 \pm .001$ \\
\hline SCUT-FBP & $.893 \pm .002 \bullet$ & $.517 \pm .001$ & $.463 \pm .001$ & $.457 \pm .001$ & $.467 \pm .001$ & $.467 \pm .001$ & $.502 \pm .001 \bullet$ & $.467 \pm .000$ & $.465 \pm .001$ \\
\hline Natural Scene & $.801 \pm .002 \bullet$ & $.649 \pm .000 \bullet$ & $.667 \pm .001 \bullet$ & $.521 \pm .001 \bullet$ & $.568 \pm .001 \bullet$ & $.520 \pm .001 \bullet$ & $.696 \pm .001 \bullet$ & $.597 \pm .002 \bullet$ & $.420 \pm .001$ \\
\hline Yeast-alpha & $.945 \pm .000 \bullet$ & $.940 \pm .000 \bullet$ & $.886 \pm .000 \bullet$ & $.882 \pm .000 \bullet$ & $.925 \pm .000 \bullet$ & $.882 \pm .001 \bullet$ & $.903 \pm .000 \bullet$ & $.891 \pm .000 \bullet$ & $.787 \pm .001$ \\
\hline Yeast-cdc & $.935 \pm .000 \bullet$ & $.926 \pm .000 \bullet$ & $.825 \pm .001$ & $.830 \pm .001 \bullet$ & $.858 \pm .000 \bullet$ & $.824 \pm .001$ & $.825 \pm .000$ & $.823 \pm .001$ & $.818 \pm .001$ \\
\hline Yeast-cold & $.730 \pm .000 \bullet$ & $.733 \pm .001 \bullet$ & $.580 \pm .001$ & $.570 \pm .001 \circ$ & $.609 \pm .000 \bullet$ & $.585 \pm .001 \bullet$ & $.586 \pm .001 \bullet$ & $.580 \pm .001$ & $.575 \pm .001$ \\
\hline Yeast-diau & $.866 \pm .000 \bullet$ & $.848 \pm .001 \bullet$ & $.711 \pm .001 \bullet$ & $.688 \pm .000 \bullet$ & $.694 \pm .001 \bullet$ & $.678 \pm .000$ & $.703 \pm .001 \bullet$ & $.694 \pm .001 \bullet$ & $.665 \pm .000$ \\
\hline Yeast-dtt & $.743 \pm .001 \bullet$ & $.740 \pm .001 \bullet$ & $.641 \pm .001$ & $.637 \pm .001$ & $.651 \pm .001 \bullet$ & $.622 \pm .001$ & $.636 \pm .001$ & $.629 \pm .001$ & $.627 \pm .001$ \\
\hline Yeast-elu & $.930 \pm .000 \bullet$ & $.924 \pm .000 \bullet$ & $.917 \pm .000 \bullet$ & $.867 \pm .001 \bullet$ & $.892 \pm .000 \bullet$ & $.875 \pm .000 \bullet$ & $.903 \pm .000 \bullet$ & $.899 \pm .000 \bullet$ & $.803 \pm .000$ \\
\hline Yeast-heat & $.824 \pm .001 \bullet$ & $.827 \pm .000 \bullet$ & $.707 \pm .001 \bullet$ & $.692 \pm .001$ & $.710 \pm .001 \bullet$ & $.680 \pm .001$ & $.717 \pm .001 \bullet$ & $.703 \pm .001 \bullet$ & $.675 \pm .001$ \\
\hline Yeast-spo & $.826 \pm .001 \bullet$ & $.817 \pm .001 \bullet$ & $.548 \pm .000$ & $.565 \pm .001 \bullet$ & $.616 \pm .000 \bullet$ & $.566 \pm .001 \bullet$ & $.584 \pm .000 \bullet$ & $.548 \pm .000$ & $.547 \pm .000$ \\
\hline Yeast-spo5 & $.667 \pm .001 \bullet$ & $.637 \pm .000 \bullet$ & $.543 \pm .001$ & $.542 \pm .000$ & $.559 \pm .001$ & $.524 \pm .001$ & $.622 \pm .000 \bullet$ & $.559 \pm .001 \bullet$ & $.534 \pm .001$ \\
\hline Yeast-spoem & $.482 \pm .000 \bullet$ & $.479 \pm .001 \bullet$ & $.441 \pm .001 \bullet$ & $.410 \pm .001$ & $.409 \pm .000$ & $.401 \pm .002$ & $.439 \pm .002 \bullet$ & $.435 \pm .002 \bullet$ & $.401 \pm .000$ \\
\hline SBU_3DFE & $.818 \pm .001 \bullet$ & $.810 \pm .001 \bullet$ & $.675 \pm .001 \bullet$ & $.616 \pm .001 \bullet$ & $.633 \pm .002 \bullet$ & $.516 \pm .001 \circ$ & $.514 \pm .001 \circ$ & $.582 \pm .001$ & $.569 \pm .001$ \\
\hline Movie & $.819 \pm .000 \bullet$ & $.677 \pm .000 \bullet$ & $.423 \pm .001 \bullet$ & $.427 \pm .000 \bullet$ & $.415 \pm .000 \bullet$ & $.410 \pm .000$ & $.454 \pm .000 \bullet$ & $.416 \pm .000 \bullet$ & $.409 \pm .000$ \\
\hline Human Gene & $.986 \pm .000 \bullet$ & $.981 \pm .000 \bullet$ & $.951 \pm .001 \bullet$ & $.966 \pm .000 \bullet$ & $.977 \pm .000 \bullet$ & $.965 \pm .000 \bullet$ & $.976 \pm .000 \bullet$ & $.955 \pm .000 \bullet$ & $.927 \pm .000$ \\
\hline
\end{tabular}

Table 3: Experimental results (mean \pm std.) of LDL4C and comparing algorithms in terms of 0/1 loss on 17 real-word datasets. In addition, $\bullet / \circ$ indicates whether LDL4C is statistically superior/inferior to the comparing algorithms.

\begin{tabular}{|c|c|c|c|c|c|c|c|c|c|}
\hline Dataset & PT-Bayes & PT-SVM & AA-BP & $\mathrm{AA}-k \mathrm{NN}$ & LDL-SVR & StructRF & LDL-SCL & BFGS-LDL & LDL4C \\
\hline SJAFFE & $.8080 \pm 3 e-4 \bullet$ & $.8305 \pm 4 \mathrm{e}-4 \bullet$ & $.8188 \pm 2 \mathrm{e}-4 \bullet$ & $.7693 \pm 6 \mathrm{e}-4 \bullet$ & $.7951 \pm 1 \mathrm{e}-4 \bullet$ & $.7766 \pm 5 e-4 \bullet$ & $.8309 \pm 2 \mathrm{e}-4 \bullet$ & $.7583 \pm 6 \mathrm{e}-4$ & $.7573 \pm 4 \mathrm{e}-4$ \\
\hline $\mathrm{M}^{2} \mathrm{~B}$ & $.6438 \pm 9 \mathrm{e}-4 \bullet$ & $.5441 \pm 4 \mathrm{e}-4$ & $.5436 \pm 4 \mathrm{e}-4$ & $.5424 \pm 4 \mathrm{e}-4$ & $.5465 \pm 3 e-4$ & $.5427 \pm 6 \mathrm{e}-4$ & $.5494 \pm 4 \mathrm{e}-4$ & $.5419 \pm 6 \mathrm{e}-4$ & $.5358 \pm 6 \mathrm{e}-4$ \\
\hline SCUT-FBP & $.8905 \pm 6 \mathrm{e}-4 \bullet$ & $.5414 \pm 2 \mathrm{e}-4$ & $.5410 \pm 2 \mathrm{e}-4$ & $.5379 \pm 3 e-4$ & $.5420 \pm 2 \mathrm{e}-4$ & $.5486 \pm 3 e-4 \bullet$ & $.5494 \pm 4 \mathrm{e}-4$ & $.5426 \pm 2 \mathrm{e}-4$ & $.5414 \pm 2 \mathrm{e}-4$ \\
\hline Natural Scene & $.8013 \pm 9 \mathrm{e}-4 \bullet$ & $.6478 \pm 1 \mathrm{e}-4$ & $.6620 \pm 3 e-4 \bullet$ & $.6658 \pm 2 \mathrm{e}-4 \bullet$ & $.6400 \pm 1 \mathrm{e}-4$ & $.6200 \pm 2 \mathrm{e}-4 \circ$ & $.6648 \pm 2 \mathrm{e}-4 \bullet$ & $.6471 \pm 2 \mathrm{e}-4$ & $.6450 \pm 3 e-4$ \\
\hline Yeast-alpha & $.9444 \pm 0 \mathrm{e}-4 \bullet$ & $.9442 \pm 0 \mathrm{e}-4 \bullet$ & $.9427 \pm 0 \mathrm{e}-4$ & $.9428 \pm 0 \mathrm{e}-4$ & $.9435 \pm 0 \mathrm{e}-4 \bullet$ & $.9429 \pm 0 \mathrm{e}-4 \bullet$ & $.9429 \pm 0 \mathrm{e}-4 \bullet$ & $.9426 \pm 0 \mathrm{e}-4$ & $.9426 \pm 0 \mathrm{e}-4$ \\
\hline Yeast-cdc & $.9329 \pm 0 \mathrm{e}-4 \bullet$ & $.9320 \pm 0 \mathrm{e}-4 \bullet$ & $.9287 \pm 0 \mathrm{e}-4$ & $.9290 \pm 0 \mathrm{e}-4$ & $.9298 \pm 0 \mathrm{e}-4 \bullet$ & $.9288 \pm 0 \mathrm{e}-4$ & $.9289 \pm 0 \mathrm{e}-4 \bullet$ & $.9287 \pm 0 \mathrm{e}-4$ & $.9287 \pm 0 \mathrm{e}-4$ \\
\hline Yeast-cold & $.7465 \pm 0 \mathrm{e}-4 \bullet$ & $.7402 \pm 0 \mathrm{e}-4 \bullet$ & $.7297 \pm 0 \mathrm{e}-4$ & $.7297 \pm 0 \mathrm{e}-4$ & $.7350 \pm 0 \mathrm{e}-4 \bullet$ & $.7291 \pm 0 \mathrm{e}-4$ & $.7304 \pm 0 \mathrm{e}-4 \bullet$ & $.7297 \pm 0 \mathrm{e}-4$ & $.7296 \pm 0 \mathrm{e}-4$ \\
\hline Yeast-diau & $.8524 \pm 0 \mathrm{e}-4 \bullet$ & $.8478 \pm 0 \mathrm{e}-4 \bullet$ & $.8432 \pm 0 \mathrm{e}-4$ & $.8424 \pm 0 \mathrm{e}-4$ & $.8432 \pm 0 \mathrm{e}-4$ & $.8424 \pm 0 \mathrm{e}-4$ & $.8427 \pm 0 \mathrm{e}-4$ & $.8428 \pm 0 \mathrm{e}-4$ & $.8428 \pm 0 \mathrm{e}-4$ \\
\hline Yeast-dtt & $.7489 \pm 0 \mathrm{e}-4 \bullet$ & $.7486 \pm 0 \mathrm{e}-4 \bullet$ & $.7421 \pm 0 \mathrm{e}-4 \bullet$ & $.7415 \pm 0 \mathrm{e}-4$ & $.7429 \pm 0 \mathrm{e}-4 \bullet$ & $.7430 \pm 0 \mathrm{e}-4 \bullet$ & $.7418 \pm 0 \mathrm{e}-4$ & $.7416 \pm 0 \mathrm{e}-4$ & $.7412 \pm 0 \mathrm{e}-4$ \\
\hline Yeast-elu & $.9285 \pm 0 \mathrm{e}-4 \bullet$ & $.9277 \pm 0 \mathrm{e}-4 \bullet$ & $.9261 \pm 0 \mathrm{e}-4$ & $.9261 \pm 0 \mathrm{e}-4$ & $.9266 \pm 0 \mathrm{e}-4 \bullet$ & $.9260 \pm 0 \mathrm{e}-4$ & $.9262 \pm 0 \mathrm{e}-4$ & $.9261 \pm 0 \mathrm{e}-4$ & $.9260 \pm 0 \mathrm{e}-4$ \\
\hline Yeast-heat & $.8299 \pm 0 \mathrm{e}-4 \bullet$ & $.8309 \pm 0 \mathrm{e}-4$ & $.8238 \pm 0 \mathrm{e}-4$ & $.8237 \pm 0 \mathrm{e}-4$ & $.8250 \pm 0 \mathrm{e}-4$ & $.8234 \pm 0 \mathrm{e}-4$ & $.8250 \pm 0 \mathrm{e}-4 \bullet$ & $.8234 \pm 0 \mathrm{e}-4$ & $.8233 \pm 0 \mathrm{e}-4$ \\
\hline Yeast-spo & $.8324 \pm 0 \mathrm{e}-4 \bullet$ & $.8165 \pm 0 \mathrm{e}-4 \bullet$ & $.8101 \pm 0 \mathrm{e}-4$ & $.8109 \pm 0 \mathrm{e}-4$ & $.8137 \pm 0 \mathrm{e}-4 \bullet$ & $.8105 \pm 0 \mathrm{e}-4$ & $.8115 \pm 0 \mathrm{e}-4$ & $.8101 \pm 0 \mathrm{e}-4$ & $.8000 \pm 0 \mathrm{e}-4$ \\
\hline Yeast-spo5 & $.6601 \pm 0 \mathrm{e}-4 \bullet$ & $.6551 \pm 0 \mathrm{e}-4$ & $.6511 \pm 0 \mathrm{e}-4$ & $.6473 \pm 0 \mathrm{e}-4$ & $.6487 \pm 0 \mathrm{e}-4$ & $.6430 \pm 0 \mathrm{e}-4 \circ$ & $.6533 \pm 0 \mathrm{e}-4$ & $.6527 \pm 0 \mathrm{e}-4$ & $.6526 \pm 0 \mathrm{e}-4$ \\
\hline Yeast-spoem & $.4909 \pm 0 \mathrm{e}-4$ & $.4756 \pm 1 \mathrm{e}-4$ & $.4717 \pm 1 \mathrm{e}-4$ & $.4689 \pm 0 \mathrm{e}-4$ & $.4720 \pm 0 \mathrm{e}-4$ & $.4670 \pm 1 e-4$ & $.4713 \pm 1 \mathrm{e}-4$ & $.4705 \pm 0 \mathrm{e}-4$ & $.4700 \pm 0 \mathrm{e}-4$ \\
\hline SBU_3DFE & $.7991 \pm 1 \mathrm{e}-4 \bullet$ & $.8093 \pm 1 \mathrm{e}-4 \bullet$ & $.8041 \pm 1 \mathrm{e}-4 \bullet$ & $.7875 \pm 0 \mathrm{e}-4 \bullet$ & $.7937 \pm 0 \mathrm{e}-4 \bullet$ & $.7745 \pm 0 \mathrm{e}-4$ & $.7769 \pm 0 \mathrm{e}-4 \bullet$ & $.7746 \pm 0 \mathrm{e}-4$ & $.7734 \pm 0 \mathrm{e}-4$ \\
\hline Movie & $.8179 \pm 0 \mathrm{e}-4 \bullet$ & $.6815 \pm 0 \mathrm{e}-4 \bullet$ & $.6760 \pm 0 \mathrm{e}-4 \bullet$ & $.6781 \pm 0 \mathrm{e}-4 \bullet$ & $.6760 \pm 0 \mathrm{e}-4 \bullet$ & $.6745 \pm 0 \mathrm{e}-4$ & $.6844 \pm 0 \mathrm{e}-4 \bullet$ & $.6755 \pm 0 \mathrm{e}-4 \bullet$ & $.6743 \pm 0 \mathrm{e}-4$ \\
\hline Human Gene & $.9848 \pm 0 \mathrm{e}-4 \bullet$ & $.9834 \pm 0 \mathrm{e}-4 \bullet$ & $.9824 \pm 0 \mathrm{e}-4 \bullet$ & $.9831 \pm 0 \mathrm{e}-4 \bullet$ & $.9822 \pm 0 \mathrm{e}-4 \bullet$ & $.9626 \pm 0 \mathrm{e}-4 \bullet$ & $.9822 \pm 0 \mathrm{e}-4 \bullet$ & $.9816 \pm 0 \mathrm{e}-4$ & $.9816 \pm 0 \mathrm{e}-4$ \\
\hline
\end{tabular}

Table 4: Experimental results (mean \pm std.) of LDL4C and comparing algorithms in terms of error probability on 17 real-word datasets.In addition, $\bullet / \circ$ indicates whether LDL4C is statistically superior/inferior to the comparing algorithms.

\section{Acknowledgments}

This research was supported by the National Key Research \& Development Plan of China (No. 2017YFB1002801), the National Science Foundation of China (61622203), the Collaborative Innovation Center of Novel Software Technology and Industrialization, and the Collaborative Innovation Center of Wireless Communications Technology.

\section{A Proof for Eq. (13)}

Proof. Recall the definition of Rademacher complexity

$$
\begin{aligned}
\hat{\mathcal{R}}\left(\Pi_{1}(\mathcal{H})\right) & =\mathbb{E}_{\sigma}\left[\frac{1}{n} \sup _{\mathbf{w}_{y}, \mathbf{w}} \sum_{i=1}^{n} \frac{\exp \left(\mathbf{x}_{i} \cdot \mathbf{w}_{y}\right)}{\sum_{j} \exp \left(\mathbf{w}_{j} \cdot \mathbf{x}_{i}\right)} \sigma_{i}\right] \\
& =\mathbb{E}_{\sigma}\left[\frac{1}{n} \sup _{\mathbf{w}_{y}, \mathbf{w}_{i=1}} \sum_{i=1}^{n} \frac{\sigma_{i}}{\left.\sum_{j} \mathrm{e}^{\left(\mathbf{w}_{j}-\mathbf{w}_{y}\right) \cdot \mathbf{x}_{i}}\right]}\right. \\
& \leq \mathbb{E}_{\sigma}\left[\frac{1}{n} \sup _{\mathbf{w}_{y}, \mathbf{w}_{i, j \neq y}} \sum^{\left(\mathbf{w}_{j}-\mathbf{w}_{y}\right) \cdot \mathbf{x}_{i}} \sigma_{i}\right] \\
& \leq \sum_{j \neq y} \frac{1}{n} \mathbb{E}_{\sigma}\left[\sup _{\mathbf{w}_{y}, \mathbf{w}_{j}} \sum_{i=1}^{n} \mathrm{e}^{\left(\mathbf{w}_{j}-\mathbf{w}_{y}\right) \cdot \mathbf{x}_{i}} \sigma_{i}\right],
\end{aligned}
$$

where $\sigma_{1}, \sigma_{2}, \ldots, \sigma_{n}$ are $n$ independent random variables with $\mathbb{P}\left(\sigma_{i}=-1\right)=\mathbb{P}\left(\sigma_{i}=1\right)=1 / 2$, and $\mathbf{W}=$ $\left[\mathbf{w}_{1}, \mathbf{w}_{2}, \ldots, \mathbf{w}_{m}\right]$ with $\left\|\mathbf{w}_{j}\right\|<1$, and the third inequality is according to the 1-Lipschitzness of function $1 / x$ for $x>1$. And for each $j \neq y$,

$$
\begin{aligned}
\mathbb{E}_{\sigma}\left[\sup _{y, j} \sum_{i=1}^{n} \frac{\sigma_{i}}{n} \mathrm{e}^{\left(\mathbf{w}_{j}-\mathbf{w}_{y}\right) \mathbf{x}_{i}}\right] & \leq \mathrm{e}^{\gamma} \mathbb{E}_{\sigma}\left[\sup _{y, j} \sum_{i=1}^{n} \frac{\sigma_{i}\left(\mathbf{w}_{j}-\mathbf{w}_{y}\right) \mathbf{x}_{i}}{n}\right] \\
& \leq \frac{\gamma \mathrm{e}^{\gamma}}{\sqrt{n}},
\end{aligned}
$$

where the first inequality is according to the $\mathrm{e}^{a}$-Lipschitzness of function $\mathrm{e}^{x}$ for $x<a$, and the second one is according to [Kakade et al., 2009] by noticing that $\left\|\mathbf{w}_{j}-\mathbf{w}_{y}\right\|<2$, which concludes the proof for Eq. (13).

\section{References}

[Bartlett and Mendelson, 2003] Peter L. Bartlett and Shahar Mendelson. Rademacher and gaussian complexities: Risk bounds and structural results. J. Mach. Learn. Res., 3:463482, March 2003.

[Chen et al., 2018] Mengting Chen, Xinggang Wang, Bin Feng, and Wenyu Liu. Structured random forest for la- 
bel distribution learning. Neurocomputing, 320:171 - 182, 2018.

[Cover and Thomas, 2012] Thomas M Cover and Joy A Thomas. Elements of information theory. John Wiley \& Sons, 2012.

[Devroye et al., 1996] Luc Devroye, László Györfi, and Gábor Lugosi. A probabilistic theory of pattern recognition, volume 31. Springer Science \& Business Media, 1996.

[Gao et al., 2017] Binbin Gao, Chao Xing, Chenwei Xie, Jianxin Wu, and Xin Geng. Deep label distribution learning with label ambiguity. IEEE Transactions on Image Processing, 26(6):2825-2838, June 2017.

[Gao et al., 2018] Binbin Gao, Hongyu Zhou, Jianxin Wu, and Xin Geng. Age estimation using expectation of label distribution learning. In Proceedings of the TwentySeventh International Joint Conference on Artificial Intelligence, IJCAI-18, pages 712-718. International Joint Conferences on Artificial Intelligence Organization, 7 2018.

[Geng and Hou, 2015] Xin Geng and Peng Hou. Pre-release prediction of crowd opinion on movies by label distribution learning. In Proceedings of the 24th International Conference on Artificial Intelligence, IJCAI'15, pages 3511-3517, 2015.

[Geng et al., 2013] Xin Geng, Chao Yin, and Zhihua Zhou. Facial age estimation by learning from label distributions. IEEE Transactions on Pattern Analysis and Machine Intelligence, 35(10):2401-2412, Oct 2013.

[Geng, 2016] Xin Geng. Label distribution learning. IEEE Transactions on Knowledge and Data Engineering, 28(7):1734-1748, July 2016.

[Huo and Geng, 2017] Zengwei Huo and Xin Geng. Ordinal zero-shot learning. In Proceedings of the Twenty-Sixth International Joint Conference on Artificial Intelligence, IJCAI-17, pages 1916-1922, 2017.

[Kakade et al., 2009] Sham M Kakade, Karthik Sridharan, and Ambuj Tewari. On the complexity of linear prediction: Risk bounds, margin bounds, and regularization. In Advances in Neural Information Processing Systems, NIPS'09, pages 793-800, 2009.

[Maurer, 2016] Andreas Maurer. A vector-contraction inequality for rademacher complexities. In Algorithmic Learning Theory, pages 3-17, 2016.

[Mohri et al., 2012] Mehryar Mohri, Afshin Rostamizadeh, and Ameet Talwalkar. Foundations of machine learning. MIT press, 2012.

[Nguyen et al., 2012] Tam V. Nguyen, Si Liu, Bingbing Ni, Jun Tan, Yong Rui, and Shuicheng Yan. Sense beauty via face, dressing, and/or voice. In Proceedings of the 20th ACM International Conference on Multimedia, MM '12, pages 239-248, New York, USA, 2012. ACM.

[Nocedal and Wright, 2006] Jorge Nocedal and Stephen Wright. Numerical optimization. Springer Science \& Business Media, 2006.
[Ren and Geng, 2017] Yi Ren and Xin Geng. Sense beauty by label distribution learning. In Proceedings of the Twenty-Sixth International Joint Conference on Artificial Intelligence, IJCAI-17, pages 2648-2654, 2017.

[Shen et al., 2017] Wei Shen, Kai Zhao, Yilu Guo, and Alan L. Yuille. Label distribution learning forests. In Proceedings of the 30th International Conference on Neural Information Processing Systems, NIPS'30, pages 834843. Curran Associates, Inc., 2017.

[Wang and Geng, 2019] Jing Wang and Xin Geng. Theoretical analysis of label distribution learning (in press). In AAAI Conference on 33th AAAI Conference on Artificial Intelligence, $A A A I^{\prime} 19,2019$.

[Xie et al., 2015] Duorui Xie, Lingyu Liang, Lianwen Jin, Jie $\mathrm{Xu}$, and Mengru Li. Scut-fbp: A benchmark dataset for facial beauty perception. 2015 IEEE International Conference on Systems, Man, and Cybernetics, Oct 2015.

[Xu et al., 2018] Ning Xu, An Tao, and Xin Geng. Label enhancement for label distribution learning. In Proceedings of the Twenty-Seventh International Joint Conference on Artificial Intelligence, IJCAI-18, pages 2926-2932. International Joint Conferences on Artificial Intelligence Organization, 72018.

[Zhang and Zhou, 2014] Minling Zhang and Zhihua Zhou. A review on multi-label learning algorithms. IEEE Transactions on Knowledge and Data Engineering, 26(8):18191837, Aug 2014.

[Zhang et al., 2015] Zhaoxiang Zhang, Wang Mo, and Geng Xin. Crowd counting in public video surveillance by label distribution learning. Neurocomputing, 166(1):151-163, 2015.

[Zheng et al., 2018] Xiang Zheng, Xiuyi Jia, and Weiwei Li. Label distribution learning by exploiting sample correlations locally, 2018.

[Zhou and Zhang, 2006] Zhihua Zhou and Minling Zhang. Multi-instance multi-label learning with application to scene classification. In Proceedings of the 19th International Conference on Neural Information Processing Systems, NIPS'06, pages 1609-1616, Cambridge, MA, USA, 2006. MIT Press.

[Zhou et al., 2015] Ying Zhou, Hui Xue, and Xin Geng. Emotion distribution recognition from facial expressions. In Proceedings of the 23rd ACM International Conference on Multimedia, MM'15, pages 1247-1250, New York, NY, USA, 2015. ACM. 